\title{
Computed Tomography Prediction of Resectability of Pancreatic Adenocarcinoma Using National Comprehensive Cancer Network Criteria
}

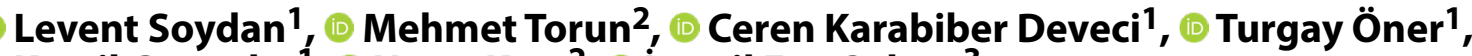 \\ Kamil Canpolat ${ }^{1}$, Umut Kına ${ }^{2}$, IIsmail Ege Subaşı ${ }^{3}$
}

${ }^{1}$ Department of Radiology, University of Health Sciences Turkey, Hamidiye Faculty of Medicine, Haydarpaşa Numune Health Application and Research Center, Istanbul, Turkey

${ }^{2}$ Department of General Surgery, University of Health Sciences Turkey, Hamidiye Faculty of Medicine, Haydarpaşa Numune Health Application and Research Center, Istanbul, Turkey

${ }^{3}$ Department of Gastroenterology, Kartal Kosuyolu High Speciality Training and Research Hospital, Istanbul, Turkey

\begin{abstract}
Introduction: We evaluated the diagnostic accuracy of computed tomography $(\mathrm{CT})$ in determining resectability of pancreatic adenocarcinoma using National Comprehensive Cancer Network (NCCN) Criteria.

Methods: Two radiologists retrospectively reviewed abdominal CT images from 36 patients with non-metastatic pancreatic adenocarcinoma who were operated on between 2018 and 20120. Based on CT findings six patients were reported as resectable, seven patients as borderline resectable, and 23 patients as unresectable using NCCN criteria for resectability. Positive predictive values for negative resection margin (R0) were assessed for CT using post-operative histopathological reports as reference standards.

Results: In 36 patients who preceded to surgery R0 resection rates were $83 \%$ (5 of 6), 71\% (5 of 7), and $60 \%$ (14 of 23 ) for resectable, borderline resectable, and unresectable disease, respectively $(p<0.001)$.

Discussion and Conclusion: CT can be used to identify patients with pancreatic cancer who can benefit from surgery with the possibility of RO resection. Our study results showed that using NCCN guidelines the diagnostic accuracy of CT to predict Ro resection can be improved.

Keywords: Computed tomography; pancreas cancer; resectability.
\end{abstract}

$P$ ancreatic adenocarcinoma is the most common form of pancreatic cancer and the fourth leading cause of cancer death in the Western world, accounting for approximately $22 \%$ of the deaths from malignant gastrointestinal neoplasms. In 2018, 458.918 new cases of pancreatic can- cer were registered worldwide, representing $2.5 \%$ of all cancers $^{[1]}$. The high mortality rate is due to the aggressive nature of the disease and due to the delayed diagnosis as the symptoms are usually vague and appear only after the disease reaches an advanced stage.

Correspondence (iletişim): Levent Soydan, M.D. Saglik Bilimleri Universitesi Hamidiye Tip Fakultesi, Haydarpasa Numune Saglik Uygulama ve Arastirma Merkezi, Radyoloji Anabilim Dali, Istanbul, Turkey

Phone (Telefon): +90 5333956490 E-mail (E-posta): levent.soydan@gmail.com

Submitted Date (Başvuru Tarihi): 15.01.2021 Accepted Date (Kabul Tarihi): 22.01.2021

Copyright 2021 Haydarpaşa Numune Medical Journal

OPEN ACCESS This is an open access article under the CC BY-NC license (http://creativecommons.org/licenses/by-nc/4.0/) 
Surgical resection of pancreatic adenocarcinoma is the only option for potential cure. However, it is estimated that only $15-20 \%$ of patients have resectable disease at presentation and even after resection the overall 5 -year survival rate remains at $15-27 \%{ }^{[2-4]}$. Treatment with neoadjuvant chemotherapy may enable achieve a negative margin resection (R0) and improve survival for patients diagnosed with early-stage pancreatic adenocarcinoma. Surgical resection should be avoided in patients who will likely not benefit from surgery ${ }^{[5,6]}$. Tumor detection and staging by assessment of resectability with diagnostic imaging are important in planning treatment. Imaging is also critical for monitoring treatment response. Although several imaging techniques, such as ultrasound (transabdominal or endoscopic ultrasound), multidetector computed tomography (MDCT), magnetic resonance imaging (MRI), and fluorodeoxyglucose positron emission tomography can be used, MDCT is the most preferred modality for the initial diagnosis and staging of pancreatic adenocarcinoma thanks to its widespread availability, superior spatial resolution, and ultra-fast coverage of a wide anatomical area that is needed for assessing of the pancreatic tumor ${ }^{[5-7]}$. In this study, we aimed to evaluate the resectability and staging of pancreatic adenocarcinoma with MDCT.

\section{Materials and Methods}

Our institutional review board approved this study. Informed patient consent was not obtained due to the retrospective nature of the study.

\section{Study Patients}

We reviewed the MDCT examinations of patients with pathologically confirmed pancreatic ductal adenocarcinoma who met the following inclusion criteria between October 2018 and October 2020: (1) Patients who had a multi-phasic CT with a pancreas protocol before surgery, (2) a time interval of less than 4 weeks between the CT scan and surgery. Among 55 eligible patients, we excluded nine patients who received neoadjuvant therapy before pancreatic surgery and ten patients who did not undergo surgery because of poor performance status, overt locally advanced or metastatic disease on imaging leaving 36 patients as our study group (33 men, three women; mean age, 63 years \pm 10 [standard deviation]; age range, 34-85 years). The data of 36 patients who underwent surgery (33 men [mean age, 62 years \pm 9 ; age range, $40-85$ years] and three women [mean age, 64 years \pm 10 ; age range, 35-81 years]) were retrospectively analyzed to evaluate the completeness of the tumor resection.

\section{Imaging Techniques}

Multiphasic CT was performed by following a pancreas protocol and using a 64-channel multidetector row CT scanner (Optima 660, GE Medical Systems, Milwaukee, USA). The scanning protocol included unenhanced and contrast material-enhanced biphasic imaging in the arterial and portal venous phases after intravenous administration of $150 \mathrm{~mL}$ of ioversol (Optiray 320; Guerbet, Villepinte, France) at a rate of $3 \mathrm{~mL} / \mathrm{s}$ using an automated power injector. Images were reconstructed at 5.0-mm thickness in the axial plane for unenhanced images and at $2.5-3.0-\mathrm{mm}$ thickness in the axial and coronal planes for arterial and portal venous phase images obtained at $20 \mathrm{~s}$ and $65 \mathrm{~s}$, respectively, following onset of contrast injection. Volume-rendered (3D) and maximal intensity projection reconstructed images for the evaluation of invasion of arterial and venous structures were generated and sent to the picture archiving and communication system for review by radiologists.

\section{Image Analysis}

Two radiologists with 17 and 4 years of experience in abdominal radiology evaluated the images. In alignment with the pancreatic cancer staging according to the National Comprehen-sive Cancer Network (NCCN) guidelines, version 1.2021 (Table 1) the degree of tumor-vascular contact was classified as no contact, abutment, encasement, occlusion, or tumor thrombosis ${ }^{[8]}$. Abutment was defined as tumor contact with no more than $180^{\circ}$ of the vessel circumference. Encasement was defined as tumor involvement with more than $180^{\circ}$ of the vessel circumference or vascular deformity suggesting invasion ${ }^{[8]}$. Hazy attenuation-vascular contact was not considered tumor-vascular contact. Lymph nodes larger than $1 \mathrm{~cm}$ in the short-axis diameter and necrotic lymph nodes were regarded as metastatic lymph nodes. Regional lymph nodes included (a) lymph nodes along the common bile duct, common hepatic artery, portomesenteric vein, and pancreaticoduodenal arcades for pancreas head cancer and (b) lymph nodes along the common hepatic artery, celiac axis, splenic artery, and splenic hilum for pancreas body or tail cancer ${ }^{[9]}$. Variant arterial anatomy, including accessory or replaced right hepatic artery and replaced common hepatic artery, and degree of tumor contact with variant arteries also were evaluated.

After determining the tumor location and size, the tumorvascular contact, adjacent organ invasion or metastatic spread were assessed and the tumor was classified as resectable, borderline resectable, or unresectable based on NCCN resectability criteria (Table 1$)^{[8]}$. Resectable disease 
Table 1. Criterıa defining resectability status at diagnosis ${ }^{a}$

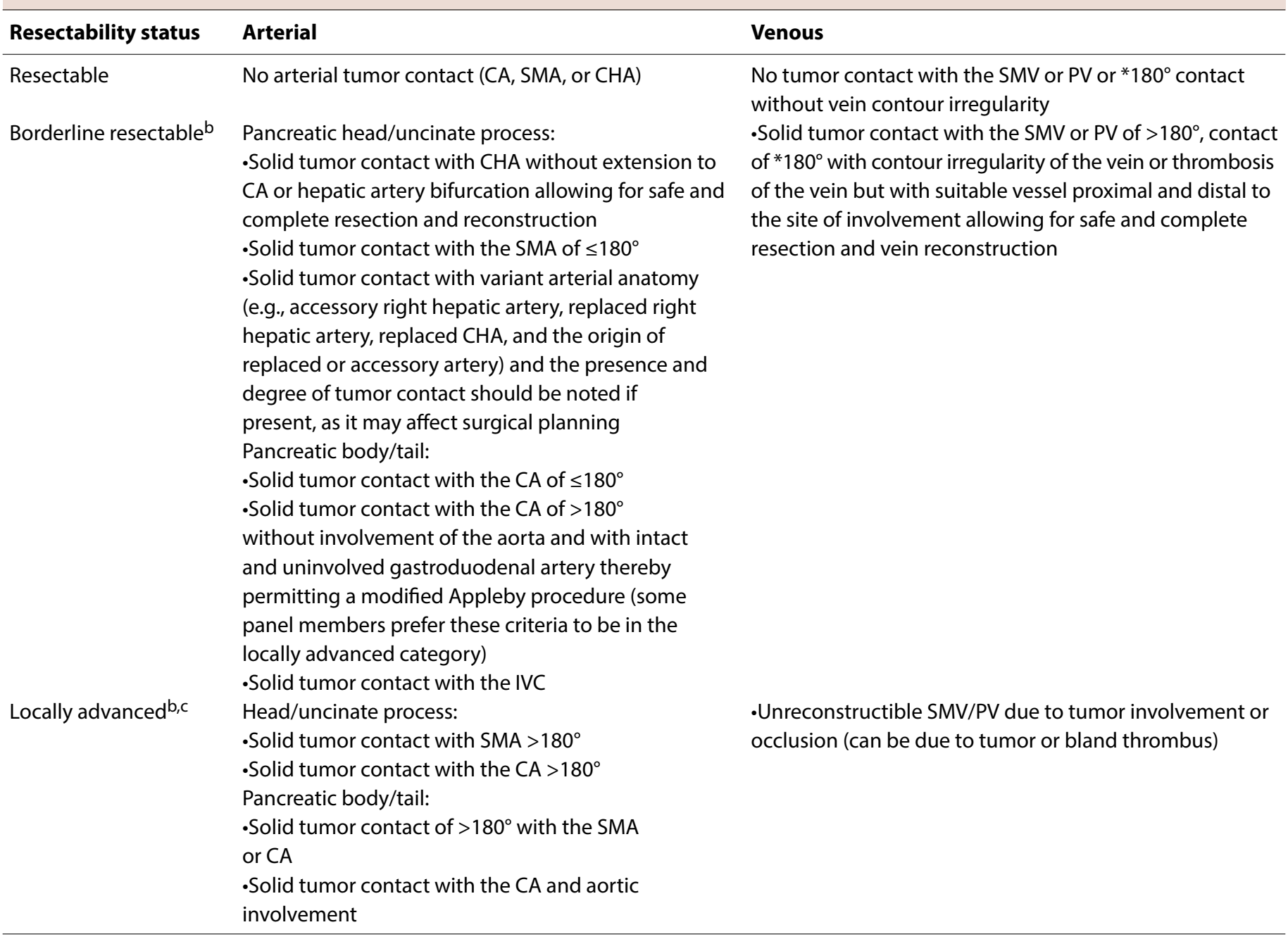

aEl Al-Hawary MM, Francis IR, Chari ST, et al. Pancreatic ductal adenocarcinoma radiology reporting template: consensus statement of the Society of Abdominal Radiology and the American Pancreatic Association. Radiology 2014; 270:248-260. bSolid tumor contact may be replaced with increased hazy density/stranding of the fat surrounding the peri-pancreatic vessels (typically seen following neoadjuvant therapy); this finding should be reported on the staging and follow-up scans. 'Distant metastasis (including non-regional lymph node metastasis), regardless of anatomic resectability, implies disease that should not be treated with upfront resection. CA: Celiac axis, SMA: Superior mesenteric artery, CHA: Common hepatic artery, SMV: Superior mesenteric vein, PV: Portal vein, IVC: Inferior vena cava.

is defined as no contact with celiac axis, SMA, CHA, SMV, or portal vein (Fig. 1). Tumor contact $\leq 180$ without vein contour irregularity is also considered resectable (Fig. 2a, b). Unresectable disease was further subdivided as locally advanced disease (pancreatic cancer without distant metastasis but with vascular invasion) (Figs. 3, 4) or M1 pancreatic cancer ${ }^{[8]}$. Radiological evaluation and assessment were done by consensus of both readers.

\section{Clinical, Surgical, Pathologic, and Follow-up Data Collection}

Therapeutic decisions were made after multidisciplinary discussion between radiologists, oncologists, and surgeons and were based on the general condition and comorbidity of patients, CT resectability, and additional imaging examinations (abdominal MRI, $\mathrm{n}=24$; combined $\mathrm{PET} / \mathrm{CT}, \mathrm{n}=11$ ). At our hospital, upfront Whipple resection without neoadjuvant therapy is the primary treatment opted in patients who are considered resectable on imaging as long as patient's general condition allows surgical intervention. The treatment for borderline resectable or locally advanced pancreatic cancer is tailored on a patient-based policy. Neoadjuvant therapy is generally saved for some borderline resectable cases and is only scarcely used at our institution. For most cases of non-metastatic borderline resectable disease, for all cases of non-metastatic locally advanced disease involving the portomesenteric veins and for selected tumors with short-segment arterial 


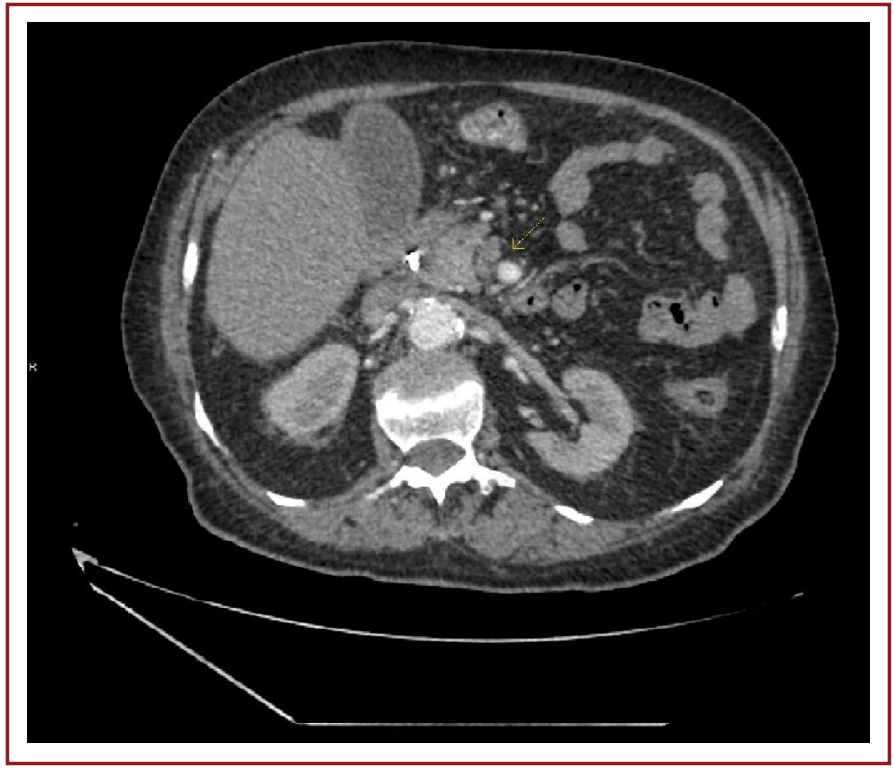

Figure 1. Adenocarcinoma in pancreatic neck with clear fat plane around superior mesenteric artery (arrow) suggesting a resectable tumor.

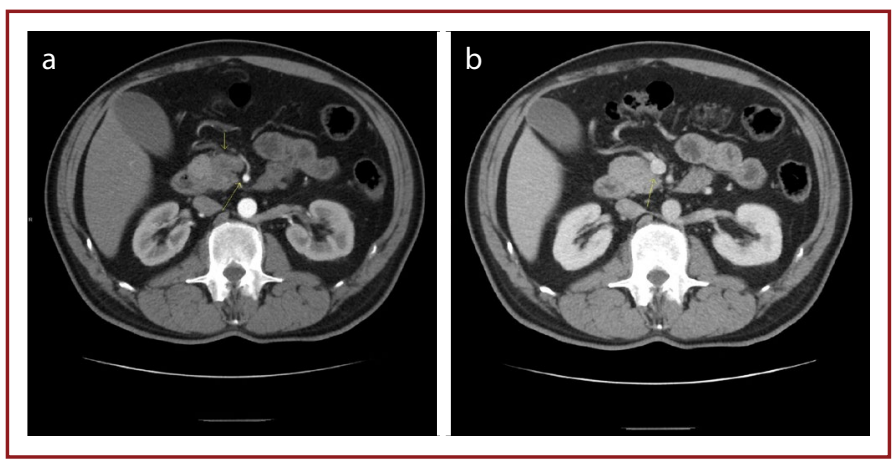

Figure 2. (a) Pancreatic head adenocarcinoma seen as hypodense area compared to lateral pancreatic parenchyma (short arrow) with a clear fat plane around celiac trunk and arising common hepatic artery (long arrow). (b) There is $\left(<180^{\circ}\right.$ abutment of the tumor with superior mesenteric vein suggesting a borderline resectable tumor (arrow).

involvement, which can be surgically re-moved with arterial decortication or short-segment arterial resection an ex-tended pancreatic surgery including the Whipple procedure and vascular reconstruction of resected vascular structures is performed. Adjuvant chemotherapy is given for these patients and for patients with metastatic pancreatic tumor at our institution.

At our hospital, surgery was performed by surgeons who have specialized at hepatobiliary and surgery. Following pancreatic resection, the pancreatic resection margin and the circumferential resection margins were marked with ink. The circumferential resection margin included the anterior and posterior surfaces of all pancreatic resec-

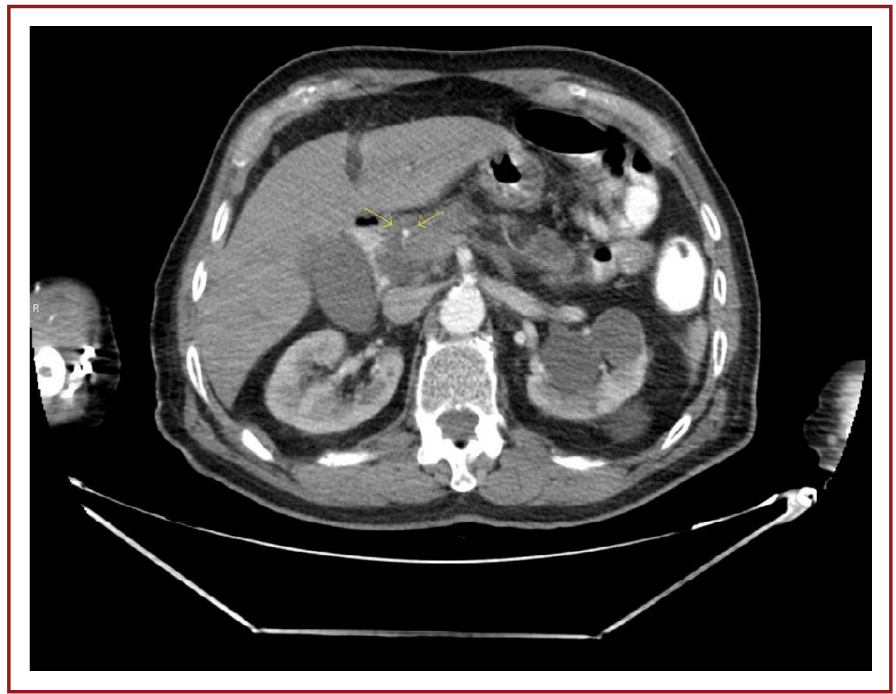

Figure 3. Circumferential encasement of common hepatic artery by pancreatic head adenocarcinoma suggesting a locally advanced unresectable tumor (arrows).

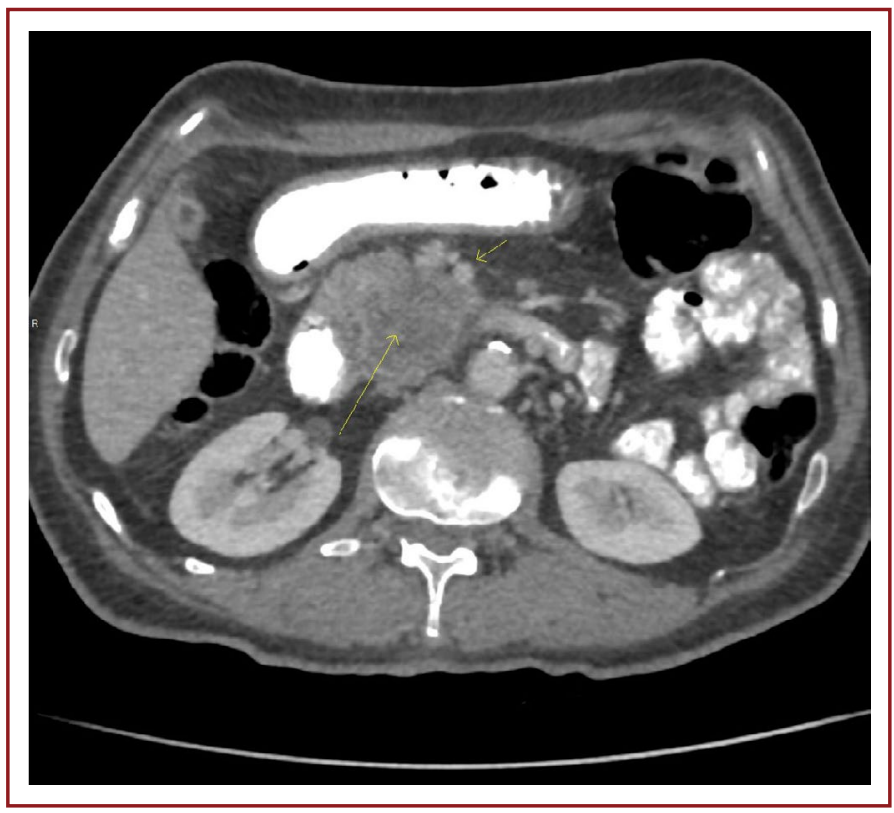

Figure 4. Pancreatic adenocarcinoma (long arrow) with abutment of superior mesenteric artery (short arrow) $>180^{\circ}$ suggesting an unresectable tumor.

tions and the common bile duct margin, uncinate process margin, superior mesenteric artery surface, and superior mesenteric vein surface for pancreaticoduodenectomy (Whipple procedure). Intraoperative pathologic examination was performed on the resected pancreas specimen. Based on British Royal College of Pathology guidelines, Ro resection was defined as complete tumor resection with a negative resection margin, $\mathrm{R} 1$ a positive resection margin with presence of tumor cells at the resection margin or within $1 \mathrm{~mm}$ of the circumferential re-section mar- 
gins, and R2 as the presence of gross residual tumor at surgery ${ }^{[10]}$.

All patient medical, surgical and pathology reports, as well as data including: Pre-operative serum cancer antigen 19-9 level; history of adjuvant chemotherapy; surgical resection method, including the presence of artery, vein, or additional organ resection; and status of resection margin (R0, R1 or R2) were retrieved. Surgical resection was classified as standard surgery (i.e., Whipple procedure or distal pancreatectomy) or extended surgery (i.e., pancreaticoduodenectomy with portomesenteric vein/artery resection for head tumors and distal pancreatectomy with splenectomy for tail tumors) according to the consensus definition of the international study group for pancreatic surgery ${ }^{[11]}$.

\section{Statistical Analysis}

$x^{2}$ tests were used for proportions and one-way analysis of variance tests or the Kruskal-Wallis test for continuous variables. All statistical analyses were performed using SPSS version 21 (IBM, Armonk, NY).

\section{Results}

\section{Patient Characteristics}

Information about our study population characteristics is summarized in Table 2 . The study population consisted of 36 patients ( 33 men and three women) with a mean age of 60 years \pm 10 (range, 31-77 years). The mean interval between the CT examination and surgery was 11 days \pm 6.3

Table 2. Characteristics of the study population

\begin{tabular}{|c|c|c|c|c|c|}
\hline \multirow[t]{2}{*}{ Characteristic } & \multirow[t]{2}{*}{ Total } & \multicolumn{3}{|c|}{ CT resectability according to NCCN criteria } & \multirow[b]{2}{*}{$\mathbf{p}$} \\
\hline & & Resectable & Borderline resectable & Unresectable & \\
\hline \multicolumn{6}{|l|}{ All patients } \\
\hline No. of patients & 55 & 8 & 16 & 31 & NA \\
\hline Age $(y)^{*}$ & $60 \pm 10$ & $63 \pm 10$ & $61 \pm 10$ & $65 \pm 10$ & 0.20 \\
\hline $\operatorname{Sex}(M / F)$ & $40 / 15$ & $7 / 1$ & $12 / 4$ & $21 / 10$ & 0.17 \\
\hline \multicolumn{6}{|l|}{ Patients who underwent surgery } \\
\hline No. of patients & 36 & 6 & 7 & 23 & $<0.001$ \\
\hline Age $(y)^{*}$ & $60 \pm 10$ & $62 \pm 9$ & $61 \pm 10$ & $65 \pm 10$ & 0.19 \\
\hline $\operatorname{Sex}(M / F)$ & $33 / 3$ & $4 / 2$ & $6 / 1$ & $23 / 0$ & 0.50 \\
\hline Tumor location in pancreas & & & & & $<0.001$ \\
\hline Head & $25(70)$ & $4(60)$ & $6(82)$ & $15(65)$ & \\
\hline Body or tail & $11(30)$ & $2(40)$ & $1(18)$ & $8(35)$ & \\
\hline Surgery & & & & & $<0.001$ \\
\hline Standard pancreaticoduodenectomy & $11(30)$ & $3(50)$ & $1(14)$ & $7(30)$ & \\
\hline Standard distal pancreatectomy & $8(22)$ & $2(33)$ & $1(14)$ & $5(22)$ & \\
\hline Extended pancreaticoduodenectomy & $13(36)$ & $1(17)$ & $2(28)$ & $10(43)$ & \\
\hline Extended distal pancreatectomy & $3(8)$ & - & $2(28)$ & $1(4)$ & \\
\hline Exploration or palliative surgery & $1(3)$ & - & $1(14)$ & - & \\
\hline Resection margin status & & & & & $<0.001$ \\
\hline Ro & $23(63)$ & $5(83)$ & $5(71)$ & $14(61)$ & \\
\hline $\mathrm{R} 1$ or $\mathrm{R} 2$ & $13(36)$ & $1(17)$ & $2(29)$ & $10(39)$ & \\
\hline Pathologic tumor differentiation & & & & & 0.48 \\
\hline Well differentiated & $3(8)$ & $1(17)$ & $1(14)$ & - & \\
\hline Moderately differentiated & $29(81)$ & $5(83)$ & $5(71)$ & $19(82)$ & \\
\hline Poorly differentiated & $4(11)$ & - & $1(14)$ & $4(18)$ & \\
\hline Pathological perineural invasion & $32(88)$ & $5(88)$ & $6(86)$ & $21(91)$ & 0.40 \\
\hline Serum CA 19-9 level (U/ml)‡ & $95(0.5,24$ 100.0) & $97.0(0.5,24100.0)$ & $105.2(0.5,24100.0)$ & $91(0.5,24$ 100.0) & 0.55 \\
\hline Adjuvant chemotherapy & $35(97)$ & $6(100)$ & $6(86)$ & $23(100)$ & \\
\hline
\end{tabular}

*Data are mean 6 standard deviation, ${ }^{* *}$ Categorized as negative margin (R0), microscopically positive margin (R1), or grossly residual tumor (R2), ${ }^{* * *}$ Data are the median, and data in parentheses are the range. Unless otherwise indicated, data are number of patients and data in parentheses are percentages. NCCN: National comprehen-sive cancer network, CA: Cancer antigen, NA: Not applicable. 
(range, 1-28 days). According to NCCN criteria, six (17\%) of 36 patients had resectable disease, seven (19\%) had borderline resectable disease, and 23 (64\%) had unresectable locally advanced disease. Surgical resection was attempted in all 36 patients. The surgical procedure included standard pancreaticoduo-denectomy $[\mathrm{n}=20(56 \%)]$; standard distal pancreatectomy $[n=4(11 \%)]$; extended pancreaticoduodenectomy $[n=11(31 \%)]$ involving portomesenteric vein resection $(n=13)$, artery resection $(n=1)$, and additional organ resection $(n=5)$ alone or in combination; and extended distal pancreatectomy $[n=1(3 \%)]$ involving portomesenteric vein and artery resection with splenectomy. In five patients, pancreatic resection was aborted due to unresectable arterial invasion $(n=4)$ or unexpected metastases in the liver and peritoneum $(n=1)$ which were detected intraoperatively.

\section{RO Resection Rate According to CT Resectability}

Of the 36 patients who underwent surgery, R0 resection was achieved in 23 (63\%). The R0 resection rate (positive predictive value) differed significantly according to resectability status as reported by CT $(\mathrm{p}<0.001)$ : It was $83 \%$ [5 of 6; $95 \%$ confidence interval $(\mathrm{Cl}): 67 \%, 78 \%$ ] in patients with resectable disease, $71 \%$ (5 of 7; $95 \% \mathrm{Cl}: 45 \%, 65 \%$ ) in patients with border-line resectable disease, and 60\% (14 of $23 ; 95 \% \mathrm{Cl}: 5 \%, 33 \%)$ in patients with locally advanced disease. R0 resection was achieved with standard surgery in $4(66 \%)$ of six patients with resectable disease and with extended surgery in six (84\%) of seven patients with borderline resectable, and $18(80 \%)$ of 23 patients with locally advanced disease. Of the 36 patients with non-metastatic pancreatic cancer, R0 resection was achieved in $63 \%$ (5 of 8) of patients with resectable disease, $31 \%$ (5 of 16) of those with borderline resectable disease, and $45 \%$ (14 of 31) of those with locally advanced disease $(p<0.001)$ (Table 2$)$.

\section{Discussion}

$\mathrm{CT}$ is a modality of choice to identify patients with a higher likelihood of margin-negative (R0) resection in pancreatic adenocarcinoma who may be candidates for upfront pancreatic resection. In these patients, neoadjuvant therapy is being increasingly used before surgery in an attempt to ensure an $\mathrm{R} 0$ resection. In locally advanced cases neoadjuvant therapy can also be used to downstage tumors to enable surgery ${ }^{[12,13]}$. According to the NCCN criteria to predict R0 resection, our results indicate that $\mathrm{CT}$ can be used to identify patients with pancreatic cancer with a likelihood of R0 resection; in our study group, the positive predictive values of CT for R0 resection were $83 \%, 71 \%$, and $60 \%$ of patients who underwent surgery for resectable, borderline resectable, and locally advanced pancreatic cancer, respectively.

We compared some previous studies in the literature that evaluated the positive predictive value of CT in the prediction of resectability of pancreatic cancer. Zamboni et al. ${ }^{[14]}$, Kaneko et al. ${ }^{[15]}$, and Lee et al. ${ }^{[16]}$ conducted studies that ignored the pathological status of the resection margin and reported positive predictive values in the range of $85 \%-89 \%$. Other studies such as those by Cassinotto et al. ${ }^{[17]}$ and Fujii et al. ${ }^{[18]}$ included the resection margin parameter and focused on the predictability of margin-negative R0 resection. Similar to the results of our study they reported slightly lower predictive values compared to studies that ignored the R0 parameter ${ }^{[17,18]}$. However, we believe that prediction of $\mathrm{R} 0$ resection rather than the resectability of the tumor should be the ultimate goal for imaging techniques as the prognostic role of the margin status is important ${ }^{[19,20]}$.

It has been suggested that some tumor parameters other than the vascular invasion findings provided by $\mathrm{CT}$ imaging may improve the prediction of R0 resection. Chiang et al. and Hong et al. reported that pancreatic cancers larger than $4 \mathrm{~cm}$ in diameter had significantly higher odds of margin-positive resection than smaller tumors thereby aiding in the prediction of unresectability ${ }^{[21,22]}$. In these studies, tumor abutment to the portomesenteric vein was significantly associated with margin-positive resection in patients with resectable pancreatic cancer suggesting that absence of these findings may be associated with higher possibility of R0 resection. The clinical implication of this could be the identification of patients who appear to have a high risk of margin-positive resection on surgery. These patients may be considered to benefit from neoadjuvant therapy in an attempt to downstage the tumor ${ }^{[23,24]}$. Due to the relatively small number of resectable patients in our study group, we could not calculate appropriate statistics to evaluate these parameters.

Our study had some limitations. First, our study group consisted only of patients who proceeded directly to surgery after imaging so patients who received neoadjuvant therapy before surgery were not included because assessment of $\mathrm{CT}$ resectability after neoadjuvant therapy is more complicated owing to post-treatment structural changes ${ }^{[25-27]}$. Second, in patients with vascular involvement pancreatic resection was performed only when venous structures were invaded. Overt signs of arterial invasion on CT precluded upfront surgery so these patients were not included. Third, abdominal MRI, PET/CT, or both were also used 
when making therapeutic decisions in most patients and this has probably prevented abortion of surgery due to unexpected detection of intraoperative metastasis. Therefore, the calculated PPV for R0 resection in our study, especially in patients with borderline resectable and locally advanced diseases, may have been overestimated. Fourth, our study may have the same limitations as all retrospective studies leading to potential weaknesses concerning the inter-reader variability and decreased consistency of study findings compared to prospective studies.

\section{Conclusion}

CT can be used to identify patients with pancreatic cancer who can benefit from surgery with the possibility of R0 resection. Our study results showed that $\mathrm{CT}$ can predict R0 resection with a positive predictive value of $75 \%$.

Peer-review: Externally peer-reviewed.

Authorship Contributions: Concept: L.S.; Design: L.S., M.T.; Data Collection or Processing: M.T., C.K.D., T.Ö., K.C.; Analysis or Interpretation: L.S., C.K.D., T.Ö., K.C., U.K., I.E.S.; Literature Search: U.K., K.C., I.E.S.; Writing: L.S., M.T.

Conflict of Interest: None declared.

Financial Disclosure: The authors declared that this study received no financial support.

\section{References}

1. Bray F, Ferlay J, Soerjomataram I, Siegel RL, Torre LA, Jemal A. Global cancer statistics 2018: GLOBOCAN estimates of incidence and mortality worldwide for 36 cancers in 185 countries. CA Cancer J Clin 2018;68:394-424.

2. Bilimoria KY, Bentrem DJ, Ko CY, Stewart AK, Winchester DP, Talamonti MS. National failure to operate on early stage pancreatic cancer. Ann Surg 2007;246:173-80.

3. Gnerlich JL, Luka SR, Deshpande AD, Dubray BJ, Weir JS, Carpenter $\mathrm{DH}$, et al. Microscopic margins and patterns of treatment failure in resected pancreatic adenocarcinoma. Arch Surg 2012;147:753-60.

4. Wagner M, Redaelli C, Lietz M, Seiler CA, Friess H, Büchler MW. Curative resection is the single most important factor determining outcome in patients with pancreatic adenocarcinoma. Br J Surg 2004;91:586-94.

5. Hartwig W, Hackert T, Hinz U, Gluth A, Bergmann F, Strobel O, et al. Pancreatic cancer surgery in the new millennium: Better prediction of outcome. Ann Surg 2011;254:311-9.

6. Li D, Xie K, Wolff R, Abbruzzese JL. Pancreatic cancer. Lancet 2004;363:1049-57.

7. Callery MP, Chang KJ, Fishman EK, Talamonti MS, Traverso LW, Linehan DC. Pretreatment assessment of resectable and borderline resectable pancreatic cancer: Expert consensus statement. Ann Surg Oncol 2009;16:1727-33.
8. Tempero MA. NCCN guidelines updates: Pancreatic cancer. J Natl Compr Canc Netw 2019;17:603-5.

9. Chun YS, Pawlik TM, Vauthey JN. 8th Edition of the AJCC cancer staging manual: Pancreas and hepatobiliary cancers. Ann Surg Oncol 2018;25:845-7.

10. Pathologists TRCo. Standards and datasets for reporting cancers. In: Dataset for Histo-Pathological Reporting of Carcinomas of the Pancreas, Ampulla of Vater and Common Bile Duct. 2nd ed. London, England: The Royal College of Pathologists; 2017.

11. Hartwig W, Vollmer CM, Fingerhut A, Yeo CJ, Neoptolemos JP, Adham $M$, et al. Extended pancreatectomy in pancreatic ductal adenocarcinoma: Definition and consensus of the international study group for pancreatic surgery (ISGPS). Surgery 2014;156:1-14.

12. Murphy JE, Wo JY, Ryan DP, Clark JW, Jiang W, Yeap BY, et al. Total neoadjuvant therapy with folfirinox in combination with losartan followed by chemoradiotherapy for locally advanced pancreatic cancer: A phase 2 clinical trial. JAMA Oncol 2019;5:1020-7.

13. Heestand GM, Murphy JD, Lowy AM. Approach to patients with pancreatic cancer without detectable metastases. J Clin Oncol 2015;33:1770-8.

14. Zamboni GA, Kruskal JB, Vollmer CM, Baptista J, Callery MP, Raptopoulos VD. Pancreatic adenocarcinoma: Value of multidetector CT angiography in preoperative evaluation. Radiology 2007;245:770-8.

15. Kaneko OF, Lee DM, Wong J, Kadell BM, Reber HA, Lu DS, et al. Performance of multidetector computed tomographic angiography in determining surgical resectability of pancreatic head adenocarcinoma. J Comput Assist Tomogr 2010;34:7328.

16. Lee JK, Kim AY, Kim PN, Lee MG, Ha HK. Prediction of vascular involvement and resectability by multidetector-row $C T$ versus MR imaging with MR angiography in patients who underwent surgery for resection of pancreatic ductal adenocarcinoma. Eur J Radiol 2010;73:310-6.

17. Cassinotto C, Dohan A, Zogopoulos G, Chiche L, Laurent C, SaCunha $A$, et al. Pancreatic adenocarcinoma: $A$ simple CT score for predicting margin-positive resection in patients with resectable disease. Eur J Radiol 2017;95:33-8.

18. Fujii T, Satoi S, Yamada S, Murotani K, Yanagimoto H, Takami $\mathrm{H}$, et al. Clinical benefits of neoadjuvant chemoradiotherapy for adenocarcinoma of the pancreatic head: An observational study using inverse probability of treatment weighting. J Gastroenterol 2017;52:81-93.

19. Kang MJ, Jang JY, Kim SW. Surgical resection of pancreatic head cancer: What is the optimal extent of surgery? Cancer Lett 2016;382:259-65.

20. Yamamoto T, Yagi S, Kinoshita H, Sakamoto Y, Okada K, Uryuhara $\mathrm{K}$, et al. Long-term survival after resection of pancreatic cancer: A single-center retrospective analysis. World J Gastroenterol 2015;21:262-8.

21. Chiang $\mathrm{KC}$, Lee $\mathrm{CH}$, Yeh $\mathrm{CN}$, Ueng $\mathrm{SH}, \mathrm{Hsu} J \mathrm{~J}$, Yeh TS, et al. $\mathrm{A}$ 
novel role of the tumor size in pancreatic cancer as an ancillary factor for predicting resectability. J Cancer Res Ther 2014;10:142-6.

22. Hong SB, Lee SS, Kim JH, Kim HJ, Byun JH, Hong SM, et al. Pancreatic cancer CT: Prediction of resectability according to NCCN criteria. Radiology 2018;289:710-8.

23. Maeda S, Moore AM, Yohanathan L, Hata T, Truty MJ, Smoot $\mathrm{RL}$, et al. Impact of resection margin status on survival in pancreatic cancer patients after neoadjuvant treatment and pancreatoduodenectomy. Surgery 2020;167:803-11.

24. Barnes CA, Chavez MI, Tsai S, Aldakkak M, George B, Ritch PS, et al. Survival of patients with borderline resectable pancreatic cancer who received neoadjuvant therapy and surgery.
Surgery 2019;166:277-85.

25. Ferrone CR, Marchegiani G, Hong TS, Ryan DP, Deshpande $\mathrm{V}, \mathrm{McDonnell} \mathrm{El}$, et al. Radiological and surgical implications of neoadjuvant treatment with FOLFIRINOX for locally advanced and borderline resectable pancreatic cancer. Ann Surg 2015;261:12-7.

26. Cassinotto C, Cortade J, Belleannée G, Lapuyade B, Terrebonne $E$, Vendrely $V$, et al. An evaluation of the accuracy of CT when determining resectability of pancreatic head adenocarcinoma after neoadjuvant treatment. Eur J Radiol 2013;82:589-93.

27. Zins M, Matos C, Cassinotto C. Pancreatic adenocarcinoma staging in the era of preoperative chemotherapy and radiation therapy. Radiology 2018;287:374-90. 